\title{
High-resolution microultrasound imaging for bladder cancer: the birth of a new diagnostic tool?
}

\author{
Marta Bottero ${ }^{1}$, Emanuele Ali ${ }^{1}$, Chiara Demofonti ${ }^{1}$, Andrea Lancia ${ }^{2}$, Riccardo Santoni ${ }^{1}$, Cynthia Aristei ${ }^{3}$, \\ Gianluca Ingrosso ${ }^{3}$
}

${ }^{1}$ Radiotherapy Unit, Department of Oncology and Hematology, Tor Vergata General Hospital, Rome, Italy; ${ }^{2}$ Department of Radiation Oncology, Fondazione IRCCS San Matteo, Pavia, Italy; ${ }^{3}$ Radiation Oncology Section, Department of Surgical and Biomedical Science, University of Perugia and Perugia General Hospital, Perugia, Italy

Correspondence to: Gianluca Ingrosso. Department of Surgical and Biomedical Science, University of Perugia, Piazza Lucio Severi 1, 06132, Perugia, Italy. Email: ingrosso.gianluca@gmail.com.

Provenance: This is an invited article commissioned by Section Editor Xiao Li (Department of Urology, Jiangsu Cancer Hospital \& Jiangsu Institute of Cancer Research \& Nanjing Medical University Affiliated Cancer Hospital, Nanjing, China).

Comment on: Saita A, Lughezzani G, Buffi NM, et al. Assessing the Feasibility and Accuracy of High-resolution Microultrasound Imaging for Bladder Cancer Detection and Staging. Eur Urol 2019. [Epub ahead of print].

Submitted Aug 09, 2019. Accepted for publication Aug 15, 2019.

doi: $10.21037 /$ tau.2019.08.20

View this article at: http://dx.doi.org/10.21037/tau.2019.08.20

Historically bladder cancer (BC) has been diagnosed with transurethral resection (TUR) (1), which is the gold standard, and imaging was not considered very useful to assess the invasiveness of BC. Computed tomography urography is generally used for BC staging but has a poor spatial resolution and provides only few details about tumor invasion into the muscolaris propria. Magnetic resonance imaging (MRI), which has a high resolution for soft tissues, might be a useful tool to assess local invasiveness. In fact, in 2018, Panebianco et al. proposed a five-point score named Vesical Imaging-Reporting And Data System (VI-RADS) based on T2-weighted, diffusion-weighted, and dynamic contrast enhancement MRI sequences (2). It represents a precious tool to help discriminate between non-muscleinvasive BC (NMIBC) and muscle-invasive BC (MIBC). However, MRI has several limitations preventing its regular use such as the high cost and the risk of overstaging caused by tumor-associated fibrosis that can be difficult to discriminate from the low signal intensity of the muscolaris propria. To overcome these limitations, Saita and colleagues decided to assess feasibility and accuracy of an imaging technique for the diagnosis of $\mathrm{BC}$, that could provide realtime evaluation, and concurrently could be cost-effective, noninvasive and accurate (3). They (3) published data of an observational prospective study evaluating the application of the $29 \mathrm{MHz}$ high-resolution microultrasound (mUS) technology in BC patients and its ability to differentiate between NMIBC and MIBC. The authors focused on four different endpoints: the feasibility of the procedure, the characterization of the three layers of bladder wall structure (the mucosa, the detrusor muscle and the adventitia), the detection of the lesions and the comparison of mUS findings with histopathological results. The first two endpoints were met; the procedure was feasible in all female patients while it failed in 2 male patients, and the 3 layers of the bladder wall were clearly distinguished in all cases (23 patients). Detection of BC was assessed only for lesions $>5 \mathrm{~mm}$ (as confirmed by the endoscopic check made after mUS). Histopathological analysis showed a good correlation for all the NMIBC, but in 2 cases mUS upstaged the lesions diagnosed as MIBC. One of the most significant advantage of mUS is the high spatial resolution down to $70 \mu \mathrm{m}$, which can provide detailed information on the three layers of the bladder wall, as they accurately showed in all 23 cases analyzed. The limitations of this procedure are the following: mUS does not allow an accurate visualization of the lateral bladder wall and therefore those lesions can remain undetected. Moreover, bladder visualization in patients presenting with a longitudinal prostate diameter longer than $5 \mathrm{~cm}$ is not accessible, and this resulted with the exclusion of two patients. Regarding the study itself, an important limitation is the low statistical significance 
of the study due to the small number of patients analyzed. Further studies with a larger population are hence necessary to standardize mUS in order to be introduced in the clinical practice. The relevance of standardizing this technique is related to the possibility of an early distinction between NMIBC and MIBC to avoid re-TUR in patients with uncertain diagnosis, which could be very crucial in some particular cases such as patients treated with anticoagulant therapy with a higher risk of bleeding.

Twenty-nine $\mathrm{MHz}$ mUS has been recently proposed to characterize prostate cancer $(\mathrm{PCa})$ with promising results in patients with suspicious lesions allowing the development of the PCa risk identification system mUS (PRI-mUS) (4). A useful and interesting study could be a comparison between mUS and other imaging techniques, in particular the mpMRI based on VI-RADS score as Lughezzani et al. (5) did in the study of PCa demonstrating the non-inferiority of mUS with respect to mpMRI in clinically significant Pca (csPCa) diagnosis. Future studies on mUS with large sample sizes evaluating the correlation with histological analysis, and comparative analyses with other imaging modalities will be crucial for the implementation of such strategy in the clinical practice.

\section{Acknowledgments}

None.

\section{Footnote}

Conflicts of Interest: The authors have no conflicts of interest to declare.

Ethical Statement: The authors are accountable for all aspects of the work in ensuring that questions related to the accuracy or integrity of any part of the work are appropriately investigated and resolved.

\section{References}

1. Alfred Witjes J, Lebret T, Compérat EM, et al. Updated 2016 EAU Guidelines on Muscle-invasive and Metastatic Bladder Cancer. Eur Urol 2017;71:462-75.

2. Panebianco V, Narumi $Y$, Altun E, et al. Multiparametric Magnetic Resonance Imaging for Bladder Cancer: Development of VI-RADS (Vesical Imaging-Reporting And Data System). Eur Urol 2018;74:294-306.

3. Saita A, Lughezzani G, Buffi NM, et al. Assessing the Feasibility and Accuracy of High-resolution Microultrasound Imaging for Bladder Cancer Detection and Staging. Eur Urol 2019. [Epub ahead of print].

4. Ghai S, Eure G, Fradet V, et al. Assessing Cancer Risk on Novel $29 \mathrm{MHz}$ Micro-Ultrasound Images of the Prostate: Creation of the Micro-Ultrasound Protocol for Prostate Risk Identification. J Urol 2016;196:562-9.

5. Lughezzani G, Saita A, Lazzeri M, et al. Comparison of the Diagnostic Accuracy of Micro-ultrasound and Magnetic Resonance Imaging/Ultrasound Fusion Targeted Biopsies for the Diagnosis of Clinically Significant Prostate Cancer. Eur Urol Oncol 2019;2:329-32 .
Cite this article as: Bottero $M$, Alì E, Demofonti C, Lancia A, Santoni R, Aristei C, Ingrosso G. High-resolution microultrasound imaging for bladder cancer: the birth of a new diagnostic tool? Transl Androl Urol 2019;8(Suppl 5):S505-S506. doi: $10.21037 /$ tau.2019.08.20 